\title{
On sharply transitive sets in $\mathrm{PG}(2, q)$
}

\author{
Alexander A. Davydov \\ Stefano Marcugini Fernanda Pambianco
}

full screen

close

\section{Abstract}

In $\operatorname{PG}(2, q)$ a point set $K$ is sharply transitive if the collineation group preserving $K$ has a subgroup acting on $K$ as a sharply transitive permutation group. By a result of Korchmáros, sharply transitive hyperovals only exist for a few values of $q$, namely $q=2,4$ and 16 . In general, sharply transitive complete arcs of even size in $\mathrm{PG}(2, q)$ with $q$ even seem to be sporadic. In this paper, we construct sharply transitive complete $6(\sqrt{q}-1)$-arcs for $q=4^{2 h+1}, h \leq 4$. As far as we are concerned, these are the smallest known complete arcs in $\mathrm{PG}\left(2,4^{7}\right)$ and in $\mathrm{PG}\left(2,4^{9}\right)$; also, 42 seems to be a new value of the spectrum of the sizes of complete arcs in $\mathrm{PG}\left(2,4^{3}\right)$. Our construction applies to any $q$ which is an odd power of 4 , but the problem of the completeness of the resulting sharply transitive arc remains open for $q \geq 4^{11}$. In the second part of this paper, sharply transitive subsets arising as orbits under a Singer subgroup are considered and their characters, that is the possible intersection numbers with lines, are investigated. Subsets of $\mathrm{PG}(2, q)$ and certain linear codes are strongly related and the above results from the point of view of coding theory will also be discussed.

Keywords : complete arcs, transitive arcs, intersection numbers

MSC 2000: 51E21

\section{Introduction}

A collineation group $G$ of $\mathrm{PG}(2, q), q$ power of a prime $p$, has a faithful action on the set of points of the plane and some point orbits of $G$ may have remarkable geometric properties. This has emerged from previous work on transitive ovals, hyperovals, arcs, $(k, n)$-arcs, blocking sets and subplanes [3, 4, 20, 21, 23, 24].

Well-known sharply transitive complete arcs other than the conics are the cyclic $(q-\sqrt{q}+1)$-arcs in $\mathrm{PG}(2, q)$ for any square $q[5,13,14,19]$, the Lunelli- 


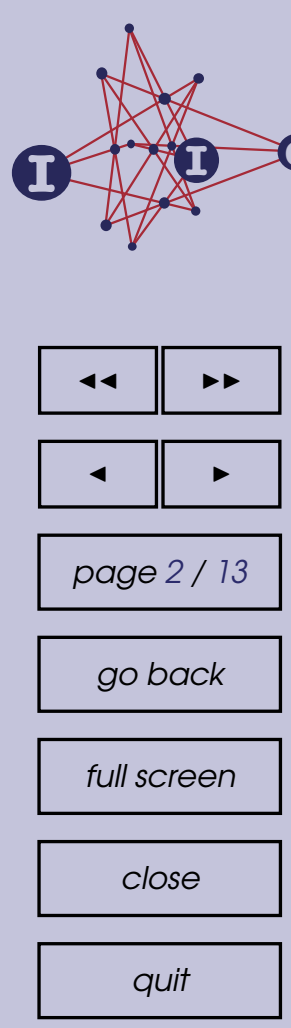

Sce hyperoval in $\mathrm{PG}(2,16)$, and the regular hyperovals in $\mathrm{PG}(2,2)$ and $\mathrm{PG}(2,4)$. Some more examples, especially for $q$ odd, are also known in the literature, see [24].

In the first part of the paper we deal with sharply transitive arcs in $\mathrm{PG}(2, q)$ for $q$ even. Apart from small $q$ 's, complete sharply transitive arcs of even size appear to be rare objects. Non-existence results are found in [20, 24, 23]. Korchmáros [20] showed that no sharply transitive hyperoval for either $q=8$ or $q \geq 32$ exists. The group $G$ cannot be both cyclic and linear, as proved by Storme and Van Maldeghem [24]. By a result of Storme [23], if $G$ is linear and does not fix a line, a point, a triangle, or an imaginary triangle (that is, a triangle in $\mathrm{PG}\left(2, q^{3}\right)$ ), then the size of the sharply transitive arc is in $\{6,18,36,72\}$.

Our contribution is on the positive side. We exhibit a complete sharply transitive $6(\sqrt{q}-1)$-arc in $\mathrm{PG}(2, q)$ for each $q=4^{2 h+1}, 1 \leq h \leq 4$. For $h=3$ and $h=4$ this seems to be the smallest known complete arc in $\mathrm{PG}\left(2,4^{2 h+1}\right)$. Also, as far as we are concerned, no other example of a complete 42 -arc in PG $(2,64)$ is known [9]. As we prove in section 2, the above arcs are members of an infinite class of sharply transitive arcs of size $6(\sqrt{q}-1)$ in $\mathrm{PG}(2, q)$, with $q=4^{2 h+1}$, $h \geq 0$. When $h=0$, then the hyperoval in $\operatorname{PG}(2,4)$ is obtained. It is still open the problem of determining whether other arcs in the family are complete.

In the second part of the paper, the case where $G$ is a subgroup of the Singer group of $\mathrm{PG}(2, q)$ is taken into consideration. Let $S$ be a Singer subgroup, that is a subgroup of the Singer group, then $|S|=\frac{q^{2}+q+1}{t}$ with $t$ a divisor of $q^{2}+q+1$.

Since the Singer group is sharply transitive on $\operatorname{PG}(2, q)$, any two point orbits under $S$ are projectively equivalent. Let $E_{t}$ be one of such orbits. For $t$ small, an important feature of $E_{t}$ is to have only a few characters. Even, $E_{t}$ may happen to have only two characters. Some sufficient conditions for this, where $q$ is a square, are due to Hamilton and Penttila [15]. In particular, this is the case when either $t$ divides $q-\sqrt{q}+1$ [10], or $q$ is a fourth power and $t$ divides $\left(q^{2}+q+1\right) /(\sqrt{q}+\sqrt[4]{q}+1)[12]$, or $p \equiv 2(\bmod 3)$ and $t=3$ [7]. For some sporadic examples with $q$ non-square, see [2]. The case $t=3$ was thoroughly investigated in $[6,8]$.

A useful tool in this investigation is the map $\phi_{t}: i \mapsto p \cdot i(\bmod t)$. Actually, as $p$ does not divide $t, \phi_{t}$ is a permutation of $\mathbb{Z}_{t}$. Hamilton and Penttila observed that $E_{t}$ has only two characters provided that $\phi_{t}$ has only two cycles, the trivial one and the other consisting of the remaining $t-1$ elements. A natural generalization which will be shown in section 3 is that $E_{t}$ has a few characters provided that $\phi_{t}$ has only a few cycles. More precisely, if $\phi_{t}$ has $r$ cycles, then $E_{t}$ has at most $r$ characters. From this, a sufficient condition on $(q, t)$ is obtained in order that $E_{t}$ has at most three characters, see Proposition 3.12. Interestingly, $E_{7}$ is always a set with at most three characters. Furthermore, when $t$ is small, 




Theorem 3.7. Let $E_{t}$ be any orbit under the action of the subgroup of the Singer group of size $\left(q^{2}+q+1\right) / t$, and let $s=s(p, t)$ be as in Definition 3.1. Then the number of characters of $E_{t}$ is at most $s(r, t)+1$.

Some lower and upper bounds on the characters of $O_{0}$ will be provided.

Lemma 3.8. Let $m_{i}$ be as in (2). Then

go back

full screen

close

quit

(i) $\sum_{i=0}^{t-1} m_{i}=q+1$;

(ii) $\sum_{i=0}^{t-1} m_{i}^{2}=\frac{q^{2}+(t+1) q+1}{t}$.

Proof. The former assertion is trivial. To prove (ii), we consider the action of $S_{n}$ on the set of lines of $\mathrm{PG}(2, q)$. For $i=0, \ldots, t-1$, let $L_{i}$ be the line orbit under $S_{n}$ containing $\sigma^{i}\left(l_{0}\right)$.

For any $u=0, \ldots, q^{2}+q$, let $s_{u}$ be such that $0 \leq s_{u} \leq t-1$ and $s_{u} \equiv-u$ $(\bmod t)$. As the collineation $\sigma^{u}$ maps the orbit $O_{s_{u}}$ on $O_{0}$,

$$
\left|\sigma^{u}\left(l_{0}\right) \cap O_{0}\right|=\left|l_{0} \cap O_{s_{u}}\right|=m_{s_{u}}
$$

holds. This proves that for any $i=0, \ldots, t-1$ the line orbit $L_{i}$ consists of lines meeting $O_{0}$ in the same number of points $m_{-i}(\bmod t)$. Then, as $O_{0}$ and $L_{i}$ have the same size, through any point $P \in O_{0}$ there pass exactly $m_{-i}(\bmod t)$ lines in $L_{i}$, each of which meets $O_{0}$ in $m_{-i}(\bmod t)$ points. Therefore, the points on $O_{0}$ can be counted as follows:

$$
\frac{q^{2}+q+1}{t}=1+\sum_{i=0}^{t-1} m_{i}\left(m_{i}-1\right)
$$

or, equivalently,

$$
\frac{q^{2}+q+1}{t}+q=\sum_{i=0}^{t-1} m_{i}^{2},
$$

whence the assertion follows.

Corollary 3.9. Let $s=s(p, t)$ be as in Definition 3.1. Let $O_{i_{1}}, \ldots, O_{i_{s}}$ be orbit representatives of the action of $\tau$ on the orbits $O_{1}, \ldots, O_{t-1}$. If $t$ is prime, then

(i) $m_{0}+\frac{t-1}{s} \sum_{j=1}^{s} m_{i_{j}}=q+1$;

(ii) $m_{0}^{2}+\frac{t-1}{s} \sum_{j=1}^{s} m_{i_{j}}^{2}=\frac{q^{2}+(t+1) q+1}{t}$. 
Theorem 3.10. Let $E_{t}$ be any orbit under the action of the subgroup of the Singer group of size $\left(q^{2}+q+1\right) / t$, and let $s=s(p, t)$ be as in Definition 3.1. If $t$ is prime, then all but at most one character $\ell$ satisfy

$$
\frac{q+1-(1+\sqrt{t s}) \sqrt{q}}{t} \leq \ell \leq \frac{q+1+(1+\sqrt{t s}) \sqrt{q}}{t}
$$

and if $\tilde{\ell}$ is the possible exception, then

$$
\frac{q+1-(t-1) \sqrt{q}}{t} \leq \tilde{\ell} \leq \frac{q+1+(t-1) \sqrt{q}}{t}
$$

Proof. Let $O_{i_{1}}, \ldots, O_{i_{s}}$ be orbit representatives of the action of $\tau$ on the orbits $O_{1}, \ldots, O_{t-1}$. Assume without loss of generality that $E_{t}=O_{0}$. We are going to prove that

$$
\frac{q+1-(t-1) \sqrt{q}}{t} \leq m_{0} \leq \frac{q+1+(t-1) \sqrt{q}}{t}
$$

and, for each $j=1, \ldots, s$,

$$
\frac{q+1-(1+\sqrt{t s}) \sqrt{q}}{t} \leq m_{i_{j}} \leq \frac{q+1+(1+\sqrt{t s}) \sqrt{q}}{t} .
$$

Let $\bar{x}$ the arithmetic mean of $\left\{m_{i_{1}}, \ldots, m_{i_{s}}\right\}$, and let $V$ be its variance. Let $\bar{y}=\bar{x}^{2}+V$. By Corollary 3.9 we have that

$$
(q+1-(t-1) \bar{x})^{2}+(t-1) \bar{y}=\frac{q^{2}+(t+1) q+1}{t} .
$$

By straightforward computation it follows that

$$
\bar{x}(2(q+1)-t \bar{x})=\frac{q^{2}+q+1}{t}+V .
$$

Then $\bar{x}(2(q+1)-t \bar{x}) \geq \frac{q^{2}+q+1}{t}$ implies that

$$
\frac{q+1-\sqrt{q}}{t} \leq \bar{x} \leq \frac{q+1+\sqrt{q}}{t} .
$$

Then (6) follows from (8), taking into account that $m_{0}=q+1-(t-1) \bar{x}$.

Also, since $\bar{x}(2(q+1)-t \bar{x}) \leq \frac{q^{2}+2 q+1}{t}$, we have that $V \leq \frac{q}{t}$. Then Chebyshev's inequality yields

$$
\left|m_{i_{j}}-\bar{x}\right| \leq \sqrt{s \frac{q}{t}}
$$

whence, taking into account (8), equation (7) follows. 
Note that equality in (5) can hold, for instance when $t=q-\sqrt{q}+1$. In this case, $E_{t}$ is a Baer subplane and $\tilde{\ell}=\sqrt{q}+1$.

The case $s(p, t)=1$ was thoroughly investigated in [15].

When $s(p, t)=2$, Theorem 3.10 can be slightly improved. Let $t=2 d+1$ and assume without loss of generality that

$$
m_{1}=\cdots=m_{d} \geq m_{d+1}=\cdots=m_{t-1} .
$$

Let $U_{1}=m_{1}+m_{d+1}$ and $U_{2}=m_{1}-m_{d+1}$. Then from Corollary 3.9 the following equality is easily obtained:

$$
2\left(q^{2}+q+1\right)+\frac{t^{2} U_{1}^{2}}{2}-2 t(q+1) U_{1}=-\frac{t U_{2}^{2}}{2},
$$

that is,

$$
t U_{2}^{2}+\left(t U_{1}-2 q-2\right)^{2}=4 q
$$

Therefore,

$$
m_{1} \leq m_{d+1}+2 \frac{\sqrt{q}}{\sqrt{t}}
$$

On the other hand (8) yields that

$$
m_{d+1} \leq-m_{1}+\frac{2}{t}(q+\sqrt{q}+1) .
$$

Then

$$
2 m_{1} \leq \frac{2}{t}(q+\sqrt{q}+1)+2 \frac{\sqrt{q}}{\sqrt{t}},
$$

and finally the following improvement of (7) is obtained.

Proposition 3.11. Assume that $s(p, t)=2$ and that $t$ is prime. Then for all but one characters $\ell$ of $E_{t}$, the following holds:

$$
\frac{q+1-(1+\sqrt{t}) \sqrt{q}}{t} \leq \ell \leq \frac{q+1+(1+\sqrt{t}) \sqrt{q}}{t} .
$$

A sufficient condition for $s(p, t)=2$ is pointed out.

Proposition 3.12. Let $t$ be a prime number such that $6 \mid(t-1)$. Let $q=p^{h}$ be such that $p \equiv \omega^{2}$ for some primitive element $\omega$ in $\mathbb{Z}_{t}$. Let $\omega^{i}$ be a primitive 6 -th roots of unity in $\mathbb{Z}_{t}$. If either

$$
h=i+d\left(\frac{t-1}{2}\right) \quad \text { or } \quad h=2 i+d\left(\frac{t-1}{2}\right)
$$

for some positive integer $d$, then both $t \mid q^{2}+q+1$ and $s(r, t)=2$ hold. 


[6] A. E. Brouwer, A series of separable designs with application to pairwise orthogonal Latin squares, European J. Combin. 1 (1980), 137-146.

[7] R. Calderbank and W. M. Kantor, The geometry of two-weight codes, Bull. London Math. Soc. 18 (1986), 97-122.

[8] J. Coykendall and J. Dover, Sets with few Intersection Numbers from Singer Subgroup Orbits, European J. Combin. 22 (2001), 455-464.

[9] A. A. Davydov, G. Faina, S. Margucini and F. Pambianco, Computer search in projective planes for the sizes of complete arcs, J. Geom. 82 (2005), 50-62.

[10] M. de Finis, On $k$-sets of type $(m, n)$ in projective planes of square order, in Finite Geometries and Designs, P.J. Cameron, J.W.P. Hirschfeld and D.R. Hughes (Eds.), London Math. Soc. Lecture Notes Ser. 49 (1981), 98-103.

[11] P. Dembowski, Finite Geometries, Springer, Berlin, 1968.

[12] M. J. de Resmini, An infinite family of type $(m, n)$ sets in $\mathrm{PG}\left(2, q^{2}\right), q$ a square, J. Geom. 20 (1983), 36-43.

[13] G. L. Ebert, Partitioning projective geometries into caps, Canad. J. Math. 37 (1985), 1163-1175.

[14] J. C. Fisher, J. W. P. Hirschfeld and J. A. Thas, Complete arcs in planes of square orders, Ann. Discrete Math. 30 (1986), 243-250.

[15] N. Hamilton and T. Penttila, Sets of Type $(a, b)$ From Subgroups of $\Gamma L\left(1, p^{R}\right)$, J. Algebraic Combin. 13 (2001), 67-76.

[16] J. W. P. Hirschfeld, Projective Geometries over Finite Fields, Clarendon Press, Oxford, 1998.

[17] J. W. P. Hirschfeld and L. Storme, The packing problem in statistics, coding theory and finite projective spaces, J. Statist. Plann. Inference 72 (1998), 355-380.

[18] W. C. Huffman, Codes and groups, Handbook of coding theory, Vol. II, 1345-1440, North-Holland, Amsterdam, 1998.

[19] B. C. Kestenband, Unital intersections in finite finite projective planes, Geom. Dedicata 11 (1981), 107-117.

[20] G. Korchmáros, Gruppi di collineazioni transitivi sui punti di una ovale $[(q+2)$-arco $]$ di $S_{2, q}, q$ pari, Atti Semin. Mat. Fis. Univ. Modena 27 (1978), 89-105. 
